Article

\title{
Biconvex Polymer Microlenses with Tunable Imaging Properties Designed by Janus Droplet Microfluidics
}

\author{
Takasi Nisisako *, Haruka Suzuki and Takeshi Hatsuzawa \\ Received: 26 August 2015 ; Accepted: 22 September 2015 ; Published: 29 September 2015 \\ Academic Editors: Andrew deMello and Xavier Casadevall i Solvas \\ Precision and Intelligence Laboratory, Tokyo Institute of Technology, R2-6, 4259 Nagatsuta-cho, Midori-ku, \\ Yokohama, Kanagawa 226-8503, Japan; suzuki.h.br@m.titech.ac.jp (H.S.); hatsuzawa.t.aa@m.titech.ac.jp (T.H.) \\ * Correspondence: nisisako.t.aa@m.titech.ac.jp; Tel./Fax: +81-45-924-5036
}

\begin{abstract}
This work presents a technique for fabricating biconvex polymer microlenses using microfluidics, and then evaluates their tunable optical properties. A glass microfluidic channel was employed to rapidly mass-produce nanoliter-sized biphasic Janus droplets, which consist of a biconvex segment of a photocurable monomer and a concave-convex segment of a non-curable silicone oil that contained a surfactant. Subsequent photopolymerization produces polymeric biconvex spherical microlenses with templated dual curvatures. By changing the flow-rate ratios of the photocurable and non-curable droplet phases in the microfluidic channel, the radii of curvature of the two lens surfaces and the thicknesses of the resultant microlenses can be varied. The resulting biconvex microlenses with different shapes were used in image projection experiments. Different magnification properties were observed, and were consistent with the properties estimated quantitatively from the geometrical parameters of the lenses.
\end{abstract}

Keywords: microfluidics; Janus droplet; biconvex microlens; interfacial tension

\section{Introduction}

Microlenses and microlens arrays are important components in photonics and optoelectronics, and are used in numerous applications-for example, in imaging systems, fiber-optic communication networks, and optical medical devices. A variety of methods, which can be divided into moldless or mold-assisted techniques, have been reported for fabricating microlenses and microlens arrays. Concerning moldless techniques, grayscale photolithography [1], thermal reflow [2], laser micromachining [3-5], inkjet printing [6], microcontact printing [7], and self-assembly [8,9] have been proposed to fabricate various polymeric, glass, silicon, and ceramic microlens arrays. However, many of these methods require sophisticated machinery, such as photo- or electron-beam lithography systems and lasers, thus, greatly limiting the high-throughput and low-cost aspects of the production of such microlenses. In addition, these methods are usually only capable of forming curvatures on one side of the substrate. On the other hand, methods based on solid master molds, which include hot embossing [10] and injection molding [11], can create microlenses with dual lens curvatures by using two precisely aligned molds. However, these methods require solid master molds that are specific to each desired shape and imaging property of a given microlens. This makes it difficult to rapidly produce microlenses with different shapes and imaging properties at low cost.

Recently, polymeric microlenses with dual lens curvatures and diameters ranging from a few hundred micrometer to a few milimeter have been prepared at fluid-fluid interfaces (liquid-liquid or liquid-air) without solid master molds. For instance, Nakazawa et al. manually dispensed a droplet of photocurable resin at the interface between hexane and water; they then photopolymerized the droplet, which exhibited a biconvex shape, to create biconvex spherical microlenses with diameters 
ranging from 0.3 to $3 \mathrm{~mm}$ [12]. More recently, Sun et al. used electrostatic forces to dispense photocurable monomer drops of controlled sizes on air-water interfaces in order to similarly create biconvex polymer parabolic lenses with controlled aperture diameters, ranging from $200 \mu \mathrm{m}$ to $4.5 \mathrm{~mm}$ [13]. A major advantage of these methods is that the size of the lenses can be tuned by adjusting the volume of the precursor droplets; moreover, their curvatures can be tuned by adjusting the balance of interfacial tensions at the interface.

To date, droplet-based microfluidic technology has been reported to fabricate polymeric lens-shaped particles such as concave-convex [14-18], biconvex [19], and biconcave [20] lenses with diameters as small as $\sim 100 \mu \mathrm{m}$. According to this technology, Janus emulsion droplets comprised of mutually immiscible photocurable and non-curable segments are produced in a microfluidic channel; the subsequent UV irradiation to the emulsion droplets yields polymerized microparticles with templated spherical convex or concave curvatures. Compared to the abovementioned methods that use bulk fluid interfaces, this microfluidic approach can easily produce even smaller lenses (e.g., $10 \mu \mathrm{m}$ or less), and at a higher throughput. Another advantage is that the curvatures can be varied by changing the volume ratio between the curable and non-curable segments of the precursor droplets, which can be controlled by adjusting the flow-rate ratios of those phases in the microfluidic channel $[15,17,19,20]$.

Recently, we have reported on optical experiments concerning biconcave microlenses [20]. The optical properties of biconvex lenses, however, have not been quantitatively analyzed yet. Accordingly, herein we report on the fabrication of biconvex polymer lenses with various shapes, and perform imaging tests to confirm their varied optical properties. Biconvex polymer microlenses with various radii of curvature were produced by changing the flow-rate ratio of the two droplet phases, using two different surfactants in the non-curable phase. Using these microlenses, we performed projection experiments to observe how varying the shape of the lens affects its magnification properties.

\section{Experimental Section}

\subsection{Microfluidic Device}

A sheath-flowing Janus droplet generator (Figure 1a) was prepared on a planar quartz glass chip by deep reactive ion etching (DRIE), as described previously [15]. Around the droplet-generating orifice, the channel width was $100 \mu \mathrm{m}$ for the three input channels and $200 \mu \mathrm{m}$ for the drain channel with a uniform depth of $100 \mu \mathrm{m}$ (Figure 1b). The microfluidic channel was used without any surface modification.

\subsection{Materials}

The external phase was a $0.3 \mathrm{wt} \%$ aqueous solution of sodium dodecyl sulfate (SDS, Wako Pure Chemicals, Tokyo, Japan). The photocurable droplet phase was 1,6-hexanediol diacrylate (dynamic viscosity $6.35 \mathrm{mPa} \cdot \mathrm{s}$, density $\rho_{\mathrm{m}}=1.02 \mathrm{~g} \cdot \mathrm{cm}^{-3}$, Shin-Nakamura Kagaku, Tokyo, Japan) containing a photoinitiator (Darocur 1173, BASF, Tokyo, Japan, $1.0 \mathrm{wt} \%$ ). In some experiments, oil-soluble dye (Oil Red O, Sigma-Aldrich, St. Louis, MO, USA) was dissolved in the monomer so as to differentiate the two droplet phases visually. For non-curable droplet phase, $10 \mathrm{cSt}$ silicone oil (SH200 10CS, $\eta_{\mathrm{s}}=9.34 \mathrm{mPa} \cdot \mathrm{s}, \rho_{\mathrm{s}}=0.934 \mathrm{~g} \cdot \mathrm{cm}^{-3}$, Dow Corning Toray, Tokyo, Japan) was used. Two surfactants (BY11-030, Dow Corning Toray; KF-6015, Shin-Etsu Chemical, Tokyo, Japan) were separately dissolved in the silicone oil to prepare $0.1 \mathrm{wt} \%$ and $1.0 \mathrm{wt} \%$ solutions, respectively. Viscosities of the liquids were measured at room temperature $(293 \mathrm{~K})$ using a rotational viscometer (B-type, Toki Sangyo, Tokyo, Japan). 

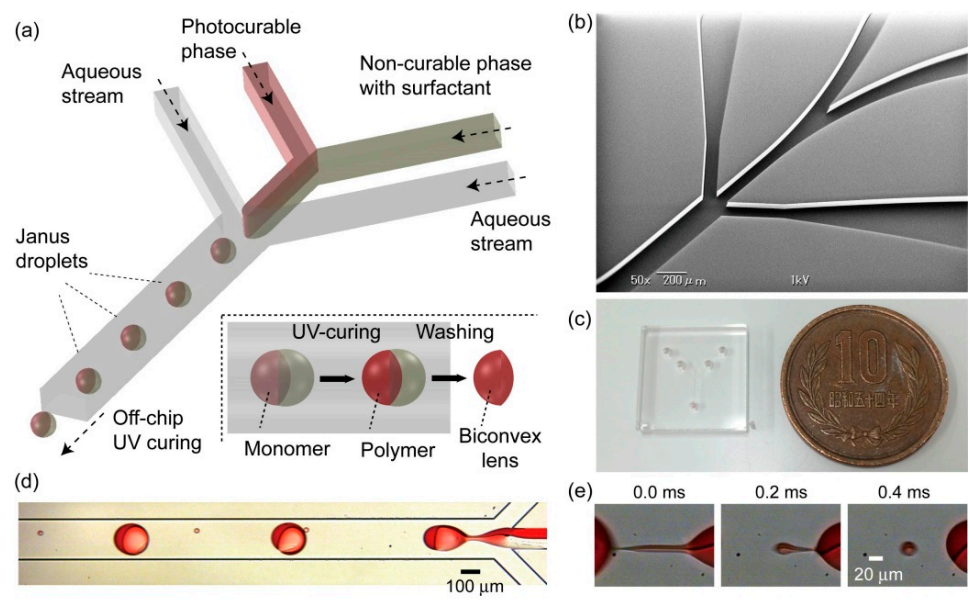

Figure 1. Microfluidic production of biconvex polymer microlenses. (a) A schematic illustration showing Janus droplet formation in a sheath-flowing channel, and the subsequent template-assisted fabrication of biconvex polymer microlenses; (b) A scanning electron microscopy (SEM) image of microgrooves dry-etched on a glass chip; (c) A glass chip (left, $15 \mathrm{~mm} \times 15 \mathrm{~mm} \times 3.5 \mathrm{~mm}$ ); (d) Formation of an array of Janus droplets. Flow rates of the disperse phase $Q_{\mathrm{d}}$ and the external aqueous phase $Q_{c}$ are $1.0\left(0.5 \mathrm{~mL} \cdot \mathrm{h}^{-1} \times 2\right)$ and $7.0 \mathrm{~mL} \cdot \mathrm{h}^{-1}\left(\right.$ i.e., $\left.3.5 \mathrm{~mL} \cdot \mathrm{h}^{-1} \times 2\right)$, respectively;

(e) Time-lapse images showing the formation of a satellite Janus droplet.

\subsection{Microfluidic Experiments}

The glass microchip (15 mm $\times 15 \mathrm{~mm} \times 3.5 \mathrm{~mm}$, Figure 1c) was assembled in a custom-made stainless steel (SUS304) holder, and was linked to syringes through poly(tetrafluoroethylene) (PTFE) tubes $(0.5 \mathrm{~mm}$ internal diameter, $1.58 \mathrm{~mm}$ external diameter). Gastight glass microsyringes (1000 series; Hamilton Company, Reno, NV, USA) were filled with the liquids, and mounted on syringe pumps (KDS Legato 200, KD Scientific, Holliston, MA, USA). Formation of Janus droplets in the microchannel was monitored and recorded by a high-speed video camera (Fastcam-1024PCI or Fastcam Mini UX100, Photron, Tokyo, Japan) mounted on an upright microscope (BX-51, Olympus, Tokyo, Japan).

\subsection{Preparation of Biconvex Polymer Microlenses}

The biconvex polymer microlenses were produced continuously via off-chip suspension polymerization under irradiation of UV light from a light source (LA-410UV, Hayashi, Japan) at the end of drain tube, at a distance of 10-15 cm. The UV intensity measured at this distance was $25-30 \mathrm{~mW} \cdot \mathrm{cm}^{-2}$ (at $365 \mathrm{~nm}$ ). Upon UV irradiation, the particles were polymerized within a few seconds. The products were filtered using a nylon mesh (grid size $57 \mu \mathrm{m} \times 57 \mu \mathrm{m}$ ), to remove smaller particles made from satellite droplets. The particles were lightly washed using acetone and ethanol under ultrasonication to remove the residues of the non-curable fluid.

\subsection{Characterization of Janus Droplets and Prepared Particles}

Both the upright optical microscope and a scanning electron microscope (SEM, VE-8800, Keyence, Osaka, Japan) were used to obtain images of the droplets and particles for characterization. An image processing software (Image Pro, Media Cybanetics, Rockville, MA, USA) was used to measure the dimensions of the prepared droplets and particles.

\subsection{Optical Experiments}

For observation through the prepared biconvex microlenses, micro patterns were laser-printed on a PET photomask film (thickness $0.175 \mathrm{~mm}, 12,700 \mathrm{dpi}$, Umino Giken, Tokyo, Japan). Quartz 
glass plates of thickness 1 or $2 \mathrm{~mm}$ were placed on the mask film and the biconvex microlenses were randomly placed on the glass slides.

\section{Results and Discussion}

\subsection{One-Step Formation of Precursor Janus Droplets}

Figure $1 \mathrm{~d}$ shows the formation of biphasic Janus droplets with the silicone oil carrying surfactant BY11-030 in a microfluidic channel at a break-off frequency of $\sim 160 \mathrm{~Hz}$. The flow rates of the photocurable and non-curable phases are both $0.5 \mathrm{~mL} \cdot \mathrm{h}^{-1}$ (i.e., $Q_{\mathrm{d}}=0.5 \mathrm{~mL} \cdot \mathrm{h}^{-1} \times 2$ ), and the flow rate of the external aqueous phase is $7.0 \mathrm{~mL} \cdot \mathrm{h}^{-1}\left(\right.$ i.e., $\left.Q_{\mathrm{c}}=3.5 \mathrm{~mL} \cdot \mathrm{h}^{-1} \times 2\right)$. By controlling the flow rates of the organic and aqueous streams appropriately in a low-capillary-number region (i.e., $10^{-3}-10^{-1}$ ), reproducible formation of primary Janus droplets was achieved. Using high-speed video microscopy, the formation of a satellite droplet measuring $\sim 20 \mu \mathrm{m}$ in diameter was observed from the stable biphasic liquid thread formed immediately before the break-off of a primary droplet (Figure 1e). Thus, biphasic Janus droplets carrying a surfactant in the non-curable phase were produced in a one-step manner, as observed previously in the formation of surfactant-free Janus droplets [15].

Figure 2a shows a $Q_{\mathrm{c}}-Q_{\mathrm{d}}$ diagram illustrating the flow conditions under which monodisperse Janus droplets can form in our device. The range of $Q_{\mathrm{c}}$ and the maximum throughput $Q_{\mathrm{d}}$ (i.e., 1.6-1.7 $\mathrm{mL} \cdot \mathrm{h}^{-1}$ ) were similar to the results previously obtained for surfactant-free Janus droplets, and also to those of a microfluidic device with a similar channel geometry $[15,19]$. Within this region, we adjusted $Q_{\mathrm{c}}$ so as to vary the detachment rate, $F_{\mathrm{b}}$, and the size of the Janus droplets. $F_{\mathrm{b}}$ varied in the range of $50-250 \mathrm{~Hz}$ (Figure $2 \mathrm{~b}$ ), whereas the equivalent diameter of the droplets, $D_{\text {eq, }}$, which is calculated from $Q_{\mathrm{d}}$ and $F_{\mathrm{b}}$, varied in the range 120-200 $\mu \mathrm{m}$ (Figure 2c). These values are consistent with our previous results for a similar microfluidic device [19].

(a)

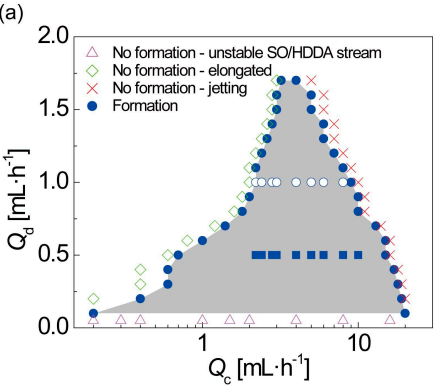

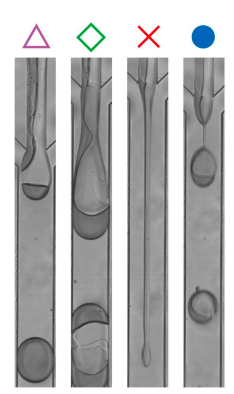

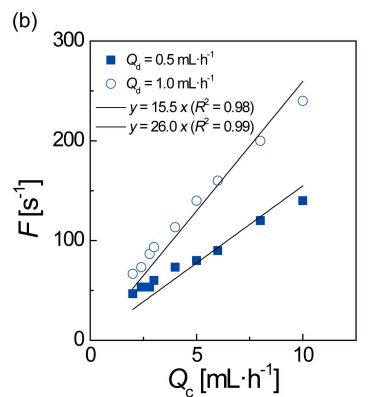

(c)

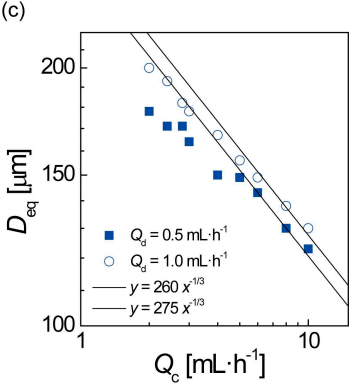

Figure 2. Hydrodynamic effects on Janus droplet formation. (a) A $Q_{c}-Q_{d}$ phase diagram illustrating the conditions under which Janus droplets can form. Open circles and solid squares represent conditions under which break-off frequencies were measured, as shown in (b); (b) Measured relationship between $Q_{\mathrm{c}}$ and the droplet break-off frequency $F$; (c) Relationship between $Q_{\mathrm{c}}$ and the equivalent diameter of a droplet in a spherical shape, $D_{\text {eq }}$, calculated from $F$ and $Q_{\mathrm{d}}$.

Characterization of the produced droplets was performed in a petri dish off the microfluidic device, thereby allowing us to study the effects of the surfactants in the non-curable silicone oil phase. For the purpose of comparison, Figure 3a shows a photomicrograph of Janus droplets produced without any surfactant in the non-curable phase. The dyed photocurable segments clearly exhibit a concave-convex shape, with a concave surface at the interface between the two droplet phases. Meanwhile, when we produced Janus droplets with the surfactant BY11-030 in the non-curable phase, the photocurable segment of the produced droplets adopted a biconvex geometry (Figure $3 \mathrm{~b}$ ). This variation in shape from concave-convex to biconvex can be explained by the shift in balance between the three interfacial tensions, which is mainly caused by the reduced interfacial tension at the interface between silicone oil and water due to the surfactant in the silicone oil segment (Figure 3c). 
The aperture diameters of the photocurable and non-curable segments were measured from the acquired images; the values of the coefficient of variation (CV) were $5.9 \%$ and $3.3 \%$, respectively (Figure 3d), which were similar to our previous results [19]. Similarly, another surfactant, KF-6015, was added in the non-curable phase and was tested under the same flow conditions. We found that the resultant droplets exhibited a Janus geometry with a similar biconvex shape; the values of the CV for the measured diameters of two segments of the droplets were $3.9 \%$ and $3.4 \%$ for photocurable and non-curable segments, respectively (see electronic supplementary information (ESI), Figure S1). Thus, both surfactants were capable of forming Janus droplets with biconvex photocurable segments.

(a)

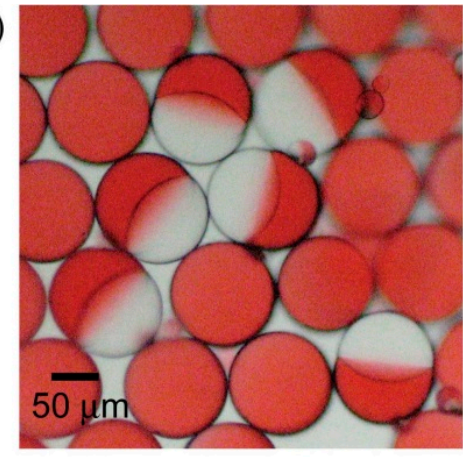

(c)

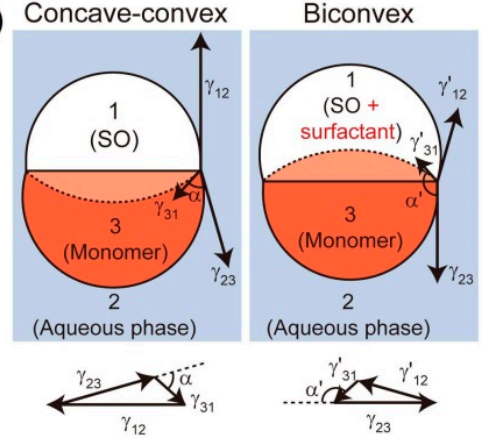

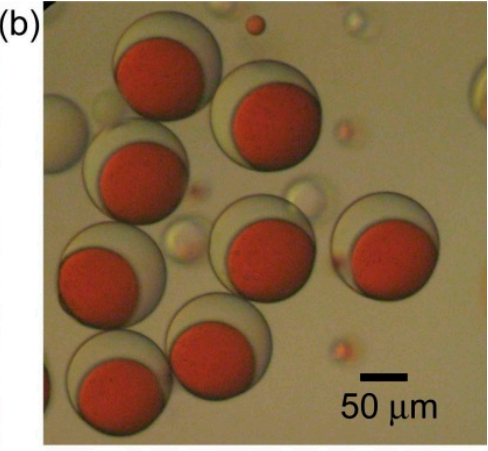

(d)

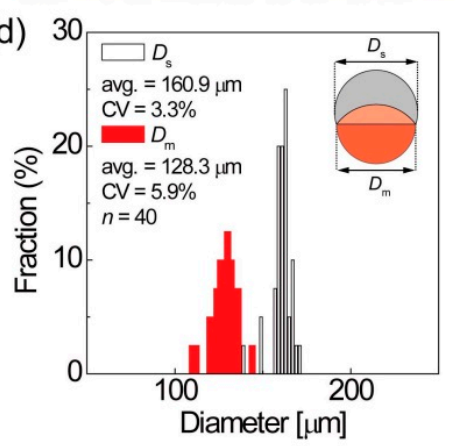

Figure 3. Janus droplets off the microfluidic chip. (a) Janus droplets without surfactant in the non-curable silicone oil (SO) segments; (b) Janus droplets with 0.1 wt \% BY11-030 surfactant in the non-curable phase; (c) Balance of three interfacial tensions and Neumann's triangles to form concave-convex and biconvex monomer segments; (d) The distributions of the sizes of two segments, $D_{\mathrm{s}}$ and $D_{\mathrm{m}}$ (see inset) of the Janus droplets in $(\mathbf{b})$.

\subsection{Photofabrication of Biconvex Polymer Lenses and Control of Their Shapes by Flow Rate Ratio}

Photopolymerization was carried out to produce biconvex microlenses from the Janus droplets with biconvex photocurable segments. In the experiments, UV light was irradiated directly to the end of the drain tube, from which precursor Janus droplets continuously emerged. Figure 4a shows a scanning electron microscopy (SEM) image of the microlenses polymerized from Janus droplets with the BY11-030 surfactant, generated at the flow-rate ratio $Q_{\mathrm{m}} / Q_{\mathrm{s}}=1 / 1$, where $Q_{\mathrm{m}}$ and $Q_{\mathrm{s}}$ are the flow rates of the monomer and silicone oil, respectively. The particles clearly have a biconvex shape. The lenses were slightly smaller than the monomer segments of the Janus droplets, possibly due to shrinkage induced by photopolymerization. The two radii of curvature of the biconvex microlenses were measured by image analysis, and were shown to be uniform (Figure $4 \mathrm{~b}$ ). At this stage, we also varied $Q_{\mathrm{m}} / Q_{\mathrm{s}}$ in the range from $1 / 2$ to $4 / 1$ in order to produce Janus droplets with different volume ratios, thereby producing biconvex particles with different shapes (Figure 4c-e). The debris and impurities on the lens surfaces can be removed further by repeating washing. Similar photopolymerization was performed on the Janus droplets with the KF-6015 surfactant, and biconvex polymer microlenses were produced with uniform curvatures (ESI, Figure S2). 
(a)

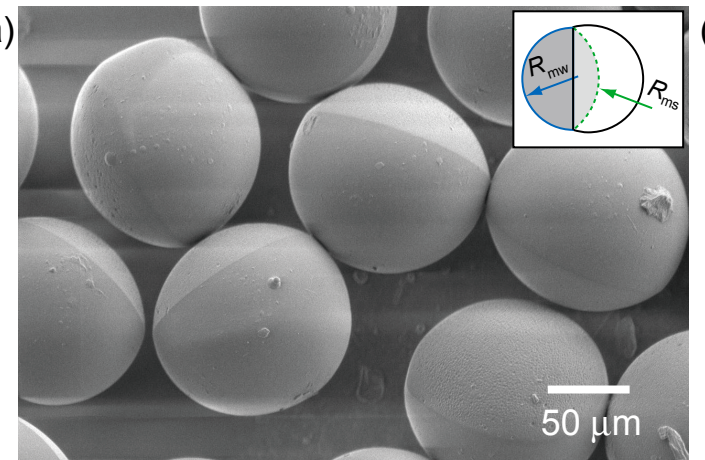

(c)

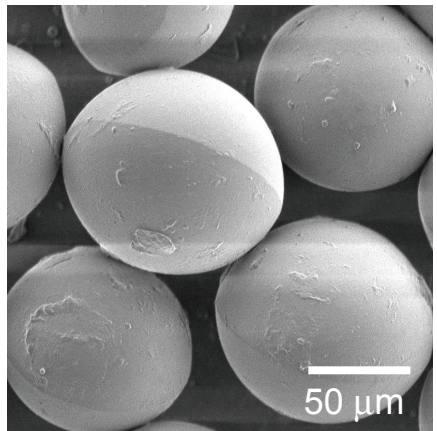

(d) (b)

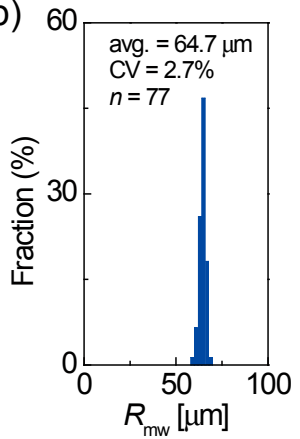

(e)
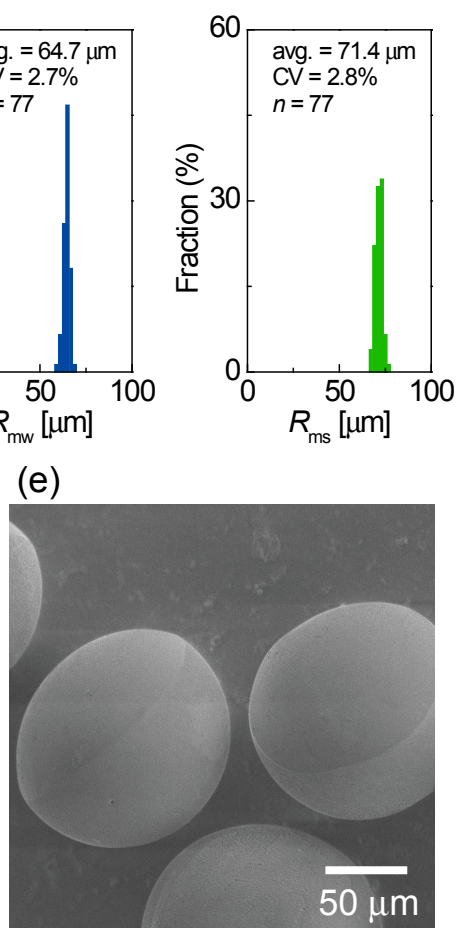

Figure 4. Polymerized biconvex particles. (a) Biconvex particles prepared with $0.1 \mathrm{wt} \%$ BY11-030, generated at the flow-rate ratio $Q_{\mathrm{m}} / Q_{\mathrm{s}}=1 / 1$, where $Q_{\mathrm{m}}$ and $Q_{\mathrm{s}}$ are the flow rates of the monomer and silicone oil, respectively; (b) The distributions of the two radii of curvature, $R_{\mathrm{mw}}$ and $R_{\mathrm{ms}}$ (see inset in (a)); (c-e) Biconvex particles prepared at (c) $Q_{\mathrm{m}} / Q_{\mathrm{s}}=1 / 2$, (d) $Q_{\mathrm{m}} / Q_{\mathrm{s}}=2 / 1$, and (e) $Q_{\mathrm{m}} / Q_{\mathrm{s}}=4 / 1$.

Figure 5a shows the relation between the volume ratio of the two segments (i.e., the flow-rate ratio of the two droplet phases) and the measured radii of curvature of the produced biconvex particles. We set the flow-rate ratio of the photocurable and non-curable phases $Q_{\mathrm{m}} / Q_{\mathrm{s}}$ as $1 / 2,1 / 1$, $2 / 1$ and $4 / 1$. As shown in the graph, all the radii of curvature increased as the ratio of the monomer phase increased. Additionally, in all flow-rate ratios studied, the radius of curvature $R_{\mathrm{ms}}$, which is the radius of the former monomer/silicone-oil interface, is always larger than $R_{\mathrm{mw}}$, which is the radius of the former monomer/water interface. For the microlenses produced with the BY11-030 surfactant, the difference between the two radii of curvature is small, whereas the microlenses produced with the KF-6015 surfactant gave rise to largely different radii of curvatures. Figure $5 \mathrm{~b}$ shows the relation between the volume ratio and the thickness of the biconvex particles. As estimated intuitively, the thickness increases as the ratio of the photocurable monomer phase increases.

The focal length $f$ of a biconvex lens can be determined by using the two radii of curvature, the thickness, and the refractive index of the lens medium according to the following lens maker's Equation [21]:

$$
\frac{1}{f}=(n-1) \times\left(\frac{1}{R_{1}}+\frac{1}{R_{2}}\right)-\left(\frac{d(n-1)^{2}}{n R_{1} R_{2}}\right)
$$

where $n$ is the refractive index of the lens medium (in this case $n=1.456$ ), $d$ is the thickness of the lens, and $R_{1}$ and $R_{2}$ are the radii of curvature of the lens where light enters and exits, respectively. Figure $5 \mathrm{c}$ shows the relation between the volume ratio and the focal length of the prepared lenses (Figure 4 and Figure S2), as calculated by Equation (1). In BY11-030 lenses, the focal length increased from 88 to $117 \mu \mathrm{m}$ when the volume ratio $Q_{\mathrm{m}} / Q_{\mathrm{s}}$ increased from $1 / 2$ to $4 / 1$. In KF-6015 lenses, the focal length similarly increased from 88 to $122 \mu \mathrm{m}$. Thus, the difference in radii of curvatures given by the two surfactants did not significantly affect the shifts in the focal length. The numerical apertures of the prepared lenses are in the range from 0.41 to 0.57 , based on NA $=D_{\mathrm{L}} / 2 f$, where NA is the numerical 
aperture and $D_{\mathrm{L}}$ is the effective lens diameter $(\sim 100 \mu \mathrm{m})$. The calculated optical resolutions of the lenses range from 0.59 to $0.82 \mu \mathrm{m}$, based on $\delta=0.61 \lambda / \mathrm{NA}$, where $\delta$ is the optical resolution of the lens, $\lambda$ is the wavelength of light $(=550 \mathrm{~nm})$.

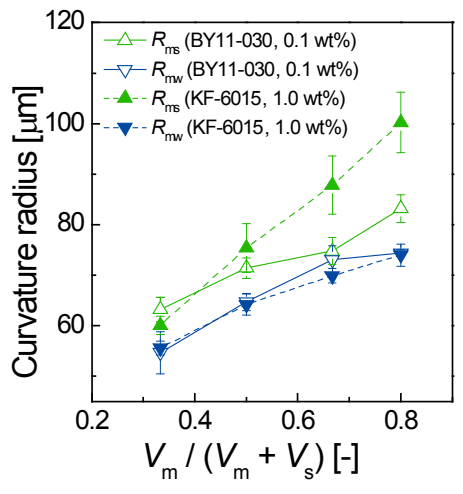

(a)

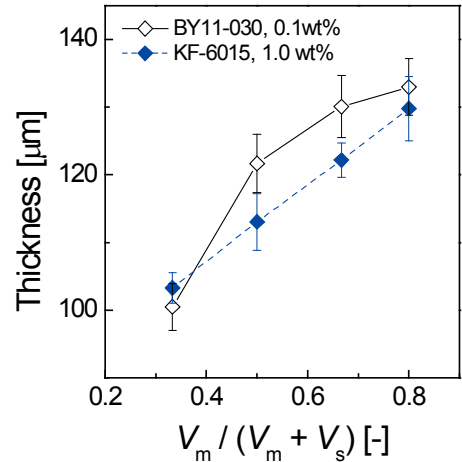

(b)

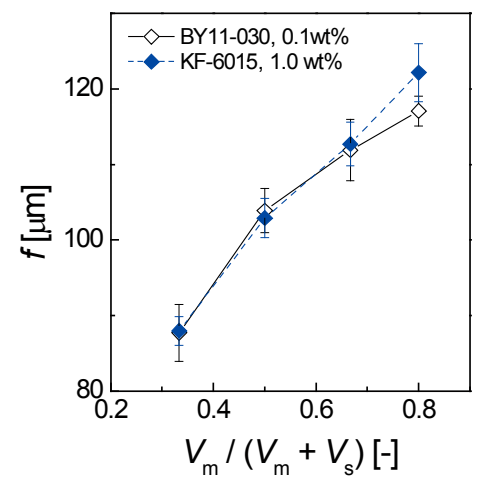

(c)

Figure 5. Effect of volume ratios of Janus segments on the measured dimensions of biconvex microlenses and calculated focal lengths. (a) Radius of curvature as a function of the volume ratio; (b) Lens thickness as a function of the volume ratio; (c) Focal length $f$ as a function of the volume ratio. $V_{\mathrm{m}}$ and $V_{\mathrm{s}}$ are the volumes of the monomer and silicone oil segments of the Janus droplets, respectively (i.e., $V_{\mathrm{m}} / V_{\mathrm{s}}=Q_{\mathrm{m}} / Q_{\mathrm{s}}$ ).

\subsection{Image Projection Experiments Using Biconvex Polymer Microlenses}

We used the prepared biconvex microlenses with different shapes in simple projection experiments to observe how the variation in shape affects the imaging properties of the lenses. Figure 6a shows the experimental setup for this imaging test. The prepared biconvex microlenses were placed on a transparent quartz glass slide (thickness of 1 or $2 \mathrm{~mm}$ ), under which a thin photomask film with microprinted patterns was inserted as the imaging object. In this setup, because the distance from the target to the lens, $S_{1}$, is well beyond the focal length of the lenses, $f(=88-122 \mu \mathrm{m})$, the real image should be projected on the phase plane at a distance $S_{2}$ from the lens in an inverted orientation (Figure 6b). Therefore, in order to identify the image inversion, we printed black micro "L"-shaped patterns (Figure 6c) on the photomask as well as mesh patterns (Figure 6d).

(a)
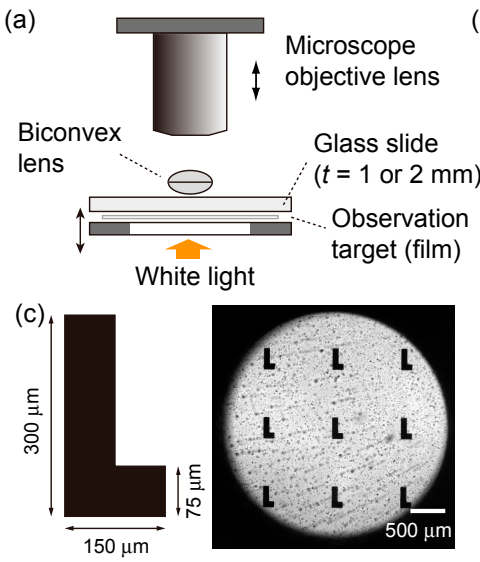

(b)
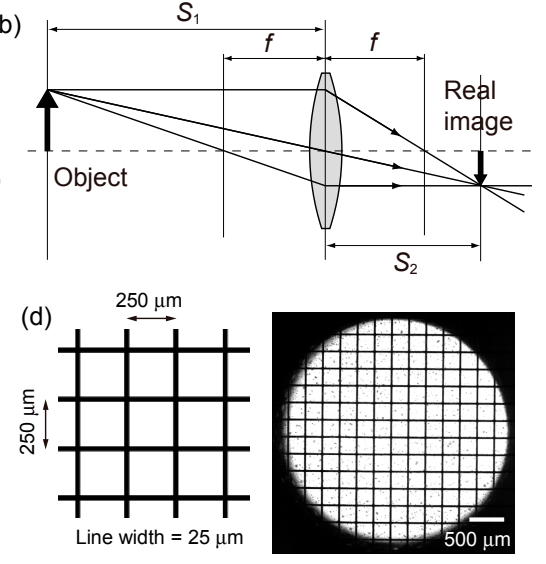

Figure 6. Schematic diagram of the image projection experiments with the prepared biconvex polymer microlenses. (a) A schematic illustration of the experimental setup; (b) The path of light through a biconvex lens when an object is placed beyond the focal point of the lens; (c) "L"-shaped patterns and (d) a mesh pattern, printed on a thin, transparent polyethylene terephthalate (PET) photomask film. 
By moving the focus of the microscope's $50 \times$ objective lens on the phase plane, which was positioned slightly above the microlenses $(\sim 100 \mu \mathrm{m})$, we were able to find the images projected by the microlenses. Figure $7 \mathrm{~b}$ shows images viewed through biconvex lenses of different curvatures on glass slides with thicknesses of 1 or $2 \mathrm{~mm}$. The miniaturized and inverted "L"-shaped pattern (as compared to the original pattern on the photomask film (Figure 7a)) was observed in all cases. In addition, we found a tendency for the magnification (i.e., the size of the projected image) to slightly increase as the volume ratio $V_{\mathrm{m}} / V_{\mathrm{s}}$ of precursor droplets increased. In addition, the images projected by the lenses at $t=2 \mathrm{~mm}$ were smaller than the images projected by the lenses at $t=1 \mathrm{~mm}$. Around the edge of the projected area, a slight distortion of the formed pattern was observed; this is probably image distortion, known as one of the monochromatic aberrations [21]. These characteristics were also observed as slight pincushion distortions when the mesh pattern was viewed through the lenses (Figure 7c,d). Due to this aberration on the edge of the lenses, it is desirable to align the object in the center of optical axis. Certainly, the microlenses also have spherical aberration along the optical axis, which is another monochromatic aberration and is typical in spherical lenses.

From Figure $6 \mathrm{~b}$, the magnification factor $M$, which is defined as the ratio of the size of the projected image to the size of the object, can be expressed by two independent parameters-the distance from the lens to the object, $S_{1}$, and the focal length, $f$-as follows:

$$
M=\frac{S_{2}}{S_{1}}=\frac{f}{S_{1}-f}
$$

this equation implies that a longer $S_{1}$ produces a more reduced image, thus explaining why the lenses at $t=2 \mathrm{~mm}$ produced smaller images compared to those of the lenses at $t=1 \mathrm{~mm}$, as shown in Figure 7. Equation (2) also explains why a biconvex lens with longer focal length results in a higher magnification when $S_{1}$ is fixed. This explains why the size of the projected image increases as the volume ratio of precursor Janus droplets increases; more specifically, as shown in Figure $5 c$, the focal length of the lenses increases as the volume ratio increases.

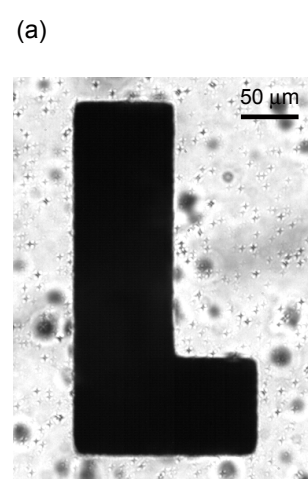

(b)
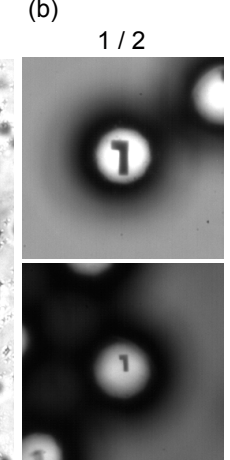

(c)

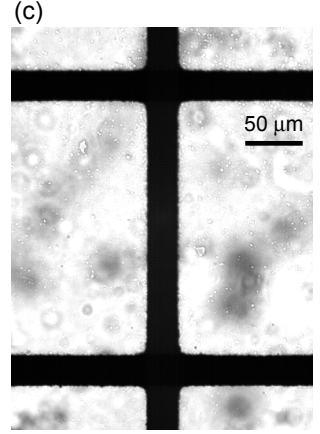

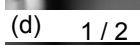

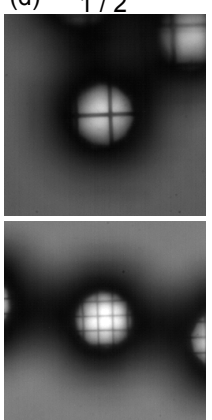

$V_{m} / V_{s}$
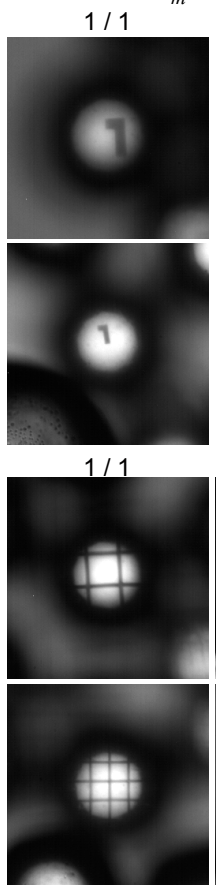

$</ 1$

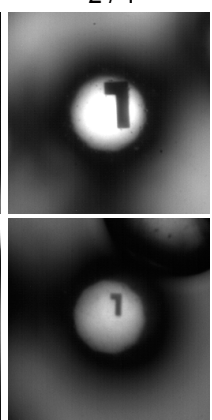

$2 / 1$

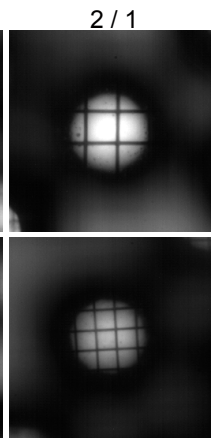

$4 / 1$
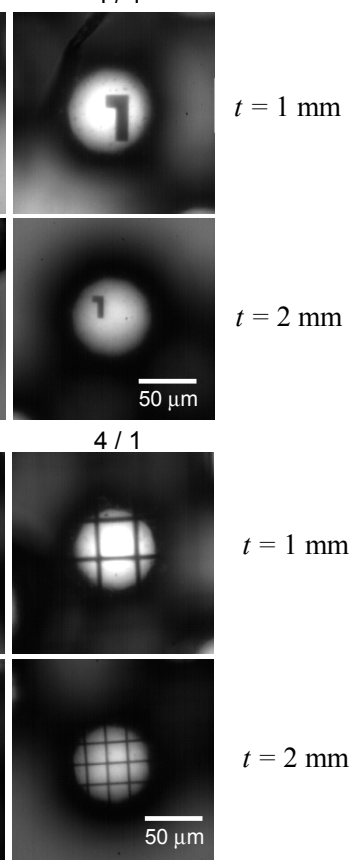

Figure 7. Observation of micro-printed patterns projected through BY11-030 biconvex microlenses positioned at two different distances. $(\mathbf{a}, \mathbf{b})$ Observation of the "L"-shaped pattern through the lenses of four different shapes; (c,d) Observation of the mesh pattern through the lenses. 
Using the biconvex lenses prepared from Janus droplets of different volume ratios, we measured the magnification $M$ through the lenses by comparing the sizes of the objects against the formed real images. Figure 8a shows the relation between the volume ratios of the precursor Janus droplets and the magnification $M$ calculated theoretically (with $f$ and $S_{1}$ using Equation (2)) and measured experimentally when the lenses are placed on the glass slide with a thickness of $1 \mathrm{~mm}$. The magnification coefficients increase in the range of $0.096-0.133$ as the volume ratio increases for BY11-030 lenses; for KF-6015 lenses (ESI, Figure S3), the magnification increases in the range of $0.096-0.142$ as the volume ratio increases. When $S_{1}=2 \mathrm{~mm}, M$ varies in the lower magnification range of 0.046-0.063 for BY11-030 lenses, and 0.046-0.068 for KF-6015 lenses; in either case, $M$ increases as the volume ratio increases (Figure 8b). For both BY11-030 and KF-6015 lenses, the theoretically estimated magnification $M_{\mathrm{t}}$ and the experimentally measured magnification $M_{\mathrm{e}}$ match each other well (Figure 8c). In this way, we demonstrated that by changing the flow-rate ratios, we were able to produce biconvex polymer microlenses with different imaging properties.
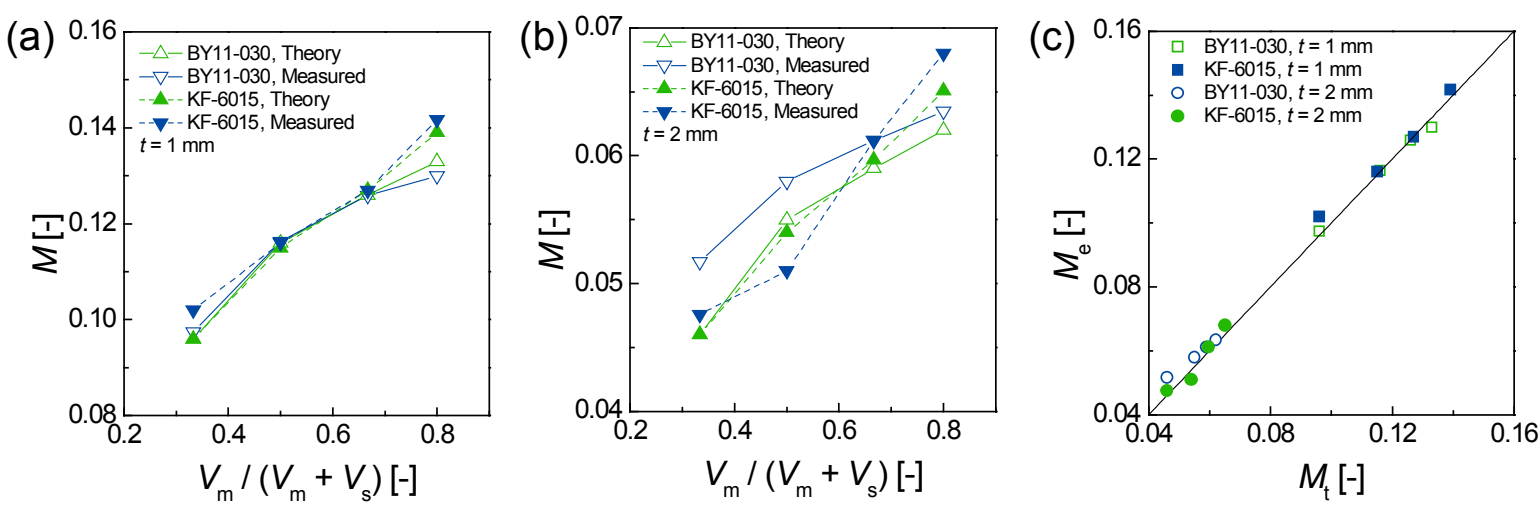

Figure 8. Magnification by the prepared biconvex microlenses. (a,b) Experimentally measured and theoretically calculated magnification of the prepared lenses as a function of the volume ratio of the precursor droplets for (a) $t=1 \mathrm{~mm}$ and (b) $t=2 \mathrm{~mm}$; (c) Measured magnification as a function of theoretical magnification.

\section{Conclusions}

We have fabricated biconvex polymer microlenses with tunable imaging properties from microfluidic Janus droplets. The radii of curvature and thicknesses of the biconvex polymer microlenses could be controlled by adjusting the volume ratios of the two droplet phases within the precursor Janus droplets; this was achieved by changing the flow-rate ratios of the two droplet phases. The projection experiments demonstrated that the prepared biconvex lenses with different shapes exhibited different optical properties, which included their focusing capability and their associated image magnification. The imaging properties of the microlenses would become further tunable by using materials with varying refractive indices as the polymerizable phase. We believe that microlenses prepared by this facile method can find wide applications in various miniature optical devices.

Supplementary Materials: Supplementary materials can be accessed at: http://www.mdpi.com/2072-666X/ 6/10/1428/s1.

Acknowledgments: This work was supported by JSPS KAKENHI Grant Numbers 19710114 and 21710124.

Author Contributions: Takasi Nisisako and Takeshi Hatsuzawa conceived and designed the experiments; Takasi Nisisako and Haruka Suzuki performed the experiments; Takasi Nisisako and Haruka Suzuki analyzed the data; Takasi Nisisako wrote the paper.

Conflicts of Interest: The authors declare no conflict of interest. 


\section{References}

1. Totsu, K.; Fujishiro, K.; Tanaka, S.; Esashi, M. Fabrication of three-dimensional microstructure using maskless gray-scale lithography. Sens. Actuators A 2006, 130-131, 387-392. [CrossRef]

2. Lin, C.-P.; Yang, H.; Chao, C.-K. Hexagonal microlens array modeling and fabrication using a thermal reflow process. J. Micromech. Microeng. 2003, 13, 775-781. [CrossRef]

3. Mihailov, S.; Lazare, S. Fabrication of refractive microlens arrays by excimer laser ablation of amorphous Teflon. Appl. Opt. 1993, 32, 6211-6218. [CrossRef] [PubMed]

4. Cheng, Y.; Tsai, H.L.; Sugioka, K.; Midorikawa, K. Fabrication of 3D microoptical lenses in photosensitive glass using femtosecond laser micromachining. Appl. Phys. A 2006, 85, 11-14. [CrossRef]

5. Shao, J.; Ding, Y.; Zhai, H.; Hu, B.; Li, X.; Tian, H. Fabrication of large curvature microlens array using confined laser swelling method. Opt. Lett. 2013, 38, 3044-3046. [CrossRef] [PubMed]

6. MacFarlane, D.L.; Narayan, V.; Tatum, J.A.; Cox, W.R.; Chen, T.; Hayes, D.J. Microjet fabrication of microlens arrays. IEEE Photon. Technol. Lett. 1994, 6, 1112-1114. [CrossRef]

7. Liu, J.; Chang, M.-J.; Ai, Y.; Zhang, H.-L.; Chen, Y. Fabrication of microlens arrays by localized hydrolysis in water droplet microreactors. ACS Appl. Mater. Interfaces 2013, 5, 2214-2219. [CrossRef]

8. Hayashi, S.; Kumamoto, Y.; Suzuki, T.; Hirai, T. Imaging by polystyrene latex particles. J. Colloid Interface Sci. 1991, 144, 538-547. [CrossRef]

9. Lu, Y.; Yin, Y.; Xia, Y. A self-assembly approach to the fabrication of patterned, two-dimensional arrays of microlenses of organic polymers. Adv. Mater. 2001, 13, 34-37. [CrossRef]

10. Ong, N.S.; Koh, Y.H.; Fu, Y.Q. Microlens array produced using hot embossing process. Microelectron. Eng. 2002, 60, 365-379. [CrossRef]

11. Lee, B.-K.; Kim, D.S.; Kwon, T.H. Replication of microlens arrays by injection molding. Microsys. Technol. 2004, 10, 531-535. [CrossRef]

12. Nakazawa, S.; Hidai, H.; Tokura, K. Photopolymerization of spherical objects using interface tension between liquids. J. Jpn. Soc. Precis. Eng. 2002, 68, 571-575. [CrossRef]

13. Sun, R.; Li, Y.; Li, L. Rapid method for fabricating polymeric biconvex parabolic lenslets. Opt. Lett. 2014, 39, 5391-5394. [CrossRef]

14. Nie, Z.; Xu, S.; Seo, M.; Lewis, P.C.; Kumacheva, E. Polymer particles with various shapes and morphologies produced in continuous microfluidic reactors. J. Am. Chem. Soc. 2005, 127, 8058-8063. [CrossRef] [PubMed]

15. Nisisako, T.; Torii, T. Formation of biphasic Janus droplets in a microfabricated channel for the synthesis of shape-controlled polymer microparticles. Adv. Mater. 2007, 19, 1489-1493. [CrossRef]

16. Pannacci, N.; Bruus, H.; Bartolo, D.; Etchart, I.; Lockhart, T.; Hennequin, Y.; Willaime, H.; Tabeling, P. Equilibrium and nonequilibrium states in microfluidic double emulsions. Phys. Rev. Lett. 2008, 101, 164502. [CrossRef] [PubMed]

17. Nisisako, T.; Hatsuzawa, T. A microfluidic cross-flowing emulsion generator for producing biphasic droplets and anisotropically shaped polymer particles. Microfluid. Nanofluid. 2010, 9, 427-437. [CrossRef]

18. Yoon, D.H.; Arakawa, T.; Go, J.S.; Shoji, S. Size controllable polymeric microlens fabrication by using a multiphase droplet including air core. In Proceedings of the 14th International Conference on miniaturized Systems for Chemistry and Life Sciences ( $\mu$ TAS 2010), Groningen, The Netherlands, 3-7 October 2010; pp. 1118-1120.

19. Nisisako, T.; Ando, T.; Hatsuzawa, T. Biconvex polymer microlenses fabricated from microfluidic Janus droplets. J. Jpn. Soc. Precis. Eng. 2013, 79, 460-466. [CrossRef]

20. Nisisako, T.; Ando, T.; Hatsuzawa, T. Capillary-assisted fabrication of biconcave polymeric microlenses from microfluidic ternary emulsion droplets. Small 2014, 10, 5116-5125. [CrossRef] [PubMed]

21. Hecht, E. Optics, 4th ed.; Addison-Wesley: San Francisco, CA, USA, 2002.

(C) 2015 by the authors; licensee MDPI, Basel, Switzerland. This article is an open access article distributed under the terms and conditions of the Creative Commons by Attribution (CC-BY) license (http://creativecommons.org/licenses/by/4.0/). 